\title{
Whole exome sequencing of adenoid cystic carcinoma
}

\author{
Philip J. Stephens, ${ }^{1}$ Helen R. Davies, ${ }^{1}$ Yoshitsugu Mitani, ${ }^{2}$ Peter Van Loo, ${ }^{1,3}$ Adam Shlien, ${ }^{1}$ \\ Patrick S. Tarpey, ${ }^{1}$ Elli Papaemmanuil, ${ }^{1}$ Angela Cheverton, ${ }^{1}$ Graham R. Bignell, ${ }^{1}$ Adam P. Butler, ${ }^{1}$ \\ John Gamble, ${ }^{1}$ Stephen Gamble, ${ }^{1}$ Claire Hardy, ${ }^{1}$ Jonathan Hinton, ${ }^{1}$ Mingming Jia, ${ }^{1}$ \\ Alagu Jayakumar, ${ }^{1}$ David Jones, ${ }^{1}$ Calli Latimer, ${ }^{1}$ Stuart McLaren, ${ }^{1}$ David J. McBride, ${ }^{1}$ \\ Andrew Menzies, ${ }^{1}$ Laura Mudie, ${ }^{1}$ Mark Maddison, ${ }^{1}$ Keiran Raine, ${ }^{1}$ Serena Nik-Zainal, ${ }^{1}$ \\ Sarah O'Meara, ${ }^{1}$ Jon W. Teague, ${ }^{1}$ Ignacio Varela, ${ }^{1}$ David C. Wedge, ${ }^{1}$ Ian Whitmore, ${ }^{1}$ \\ Scott M. Lippman, ${ }^{4}$ Ultan McDermott, ${ }^{1}$ Michael R. Stratton, ${ }^{1}$ \\ Peter J. Campbell, ${ }^{1}$ Adel K. El-Naggar, ${ }^{2}$ and P. Andrew Futreal ${ }^{1}$

\begin{abstract}
${ }^{1}$ Cancer Genome Project, Wellcome Trust Sanger Institute, Wellcome Trust Genome Campus, Hinxton, Cambridgeshire, United Kingdom. 2Department of Pathology, The University of Texas MD Anderson Cancer Center, Houston, Texas, USA. ${ }^{3}$ Human Genome Laboratory, Department of Human Genetics, VIB and KU Leuven, Leuven, Belgium. ${ }^{4}$ Department of Thoracic/Head and Neck Medical Oncology, The University of Texas MD Anderson Cancer Center, Houston, Texas, USA.
\end{abstract}

\begin{abstract}
Adenoid cystic carcinoma (ACC) is a rare malignancy that can occur in multiple organ sites and is primarily found in the salivary gland. While the identification of recurrent fusions of the MYB-NFIB genes have begun to shed light on the molecular underpinnings, little else is known about the molecular genetics of this frequently fatal cancer. We have undertaken exome sequencing in a series of 24 ACC to further delineate the genetics of the disease. We identified multiple mutated genes that, combined, implicate chromatin deregulation in half of cases. Further, mutations were identified in known cancer genes, including PIK3CA, ATM, CDKN2A, SF3B1, $S U F U, T S C 1$, and CYLD. Mutations in NOTCH1/2 were identified in 3 cases, and we identify the negative NOTCH signaling regulator, SPEN, as a new cancer gene in ACC with mutations in 5 cases. Finally, the identification of 3 likely activating mutations in the tyrosine kinase receptor FGFR2, analogous to those reported in ovarian and endometrial carcinoma, point to potential therapeutic avenues for a subset of cases.
\end{abstract}

\section{Introduction}

Adenoid cystic carcinoma (ACC) is a rare salivary gland malignancy characterized by an indolent clinical course with multiple local recurrences. Even with effective local control, the majority of patients succumb to metastatic disease within a decade. Thus, there is a pressing need for new therapies fueled by improved insights into the biology of the disease. Here we report the exome sequencing analyses of 24 cases of ACC and the elaboration of the genetics of this rare cancer.

Only a handful of point mutations in known cancer genes have been reported in ACC, including KRAS, BRAF, and TP53 (1). Recent progress has come in the identification of a recurrent translocation $\mathrm{t}(6 ; 9)(\mathrm{q} 22-23 ; \mathrm{p} 23-24)$ resulting in the fusion of the $\mathrm{v}$-myb myeloblastosis viral oncogene homolog $(M Y B)$ gene on chromosome 6 to the nuclear factor I/B (NFIB) gene on chromosome 9 in the majority of cases (2). However, the extent to which other genes are contributing to the disease and thus constitute additional targets for potential therapeutic exploitation is not well characterized.

\section{Results and Discussion}

Twenty-three pretreatment primary ACC specimens, 1 localregional lymph node metastasis (Supplemental Table 1; supplemental material available online with this article; doi:10.1172/ JCI67201DS1), and corresponding matching normal salivary gland parenchymal samples were subjected to solution phase

Authorship note: Philip J. Stephens and Helen R. Davies contributed equally to this work.

Conflict of interest: The authors have declared that no conflict of interest exists. Citation for this article: J Clin Invest. 2013;123(7):2965-2968. doi:10.1172/JCI67201. capture and next-generation sequencing of the coding exome as well as evaluation for and validation of somatic mutations as previously described (3). The case series consisted of 19 that were scored as positive for MYB activation and comprised a roughly equal mixture of cribriform and predominantly solid histological forms of ACC (Supplemental Table 1). The latter are associated with increased risk of local-regional relapse, distant metastases, and poorer overall survival (4).

Exome sequencing identified 312 somatic mutations, ranging from 2 in PD3198a to 35 in PD3181a (Supplemental Table 2), with a mean of 13 mutations per exome, lower than that reported for most adult solid tumors thus far. The mutations comprised 182 missense, 59 silent, 14 nonsense, 45 frameshift, 6 in-frame insertion/deletion, and 6 essential splice site. There was no statistically significant difference between the average numbers of somatic mutations in different histological subtypes, nor between MYB-positive and -negative cases. Recurrent losses of 1p36, 6q, 9 p, 12q were noted from SNP arrays (Supplemental Table 3 and Supplemental Figure 1), in agreement with previous work $(5,6)$. A substantial proportion of cases with likely MYB activation also demonstrated breakpoints and copy number changes on SNP6 arrays at MYB and NFIB loci (Supplemental Table 3), potentially accounting for a substantial proportion of the reported involvement of these regions.

Somatic mutations were identified in multiple known cancer genes (Figure 1) including a CDKN2A truncating frameshift mutation. Further analyses of SNP6-derived copy number data identified 3 additional cases (Supplemental Table 4) with loss of heterozygosity encompassing the CDKN2A locus where there was no apparent involvement of the nearby NFIB locus in a $M Y B$ 

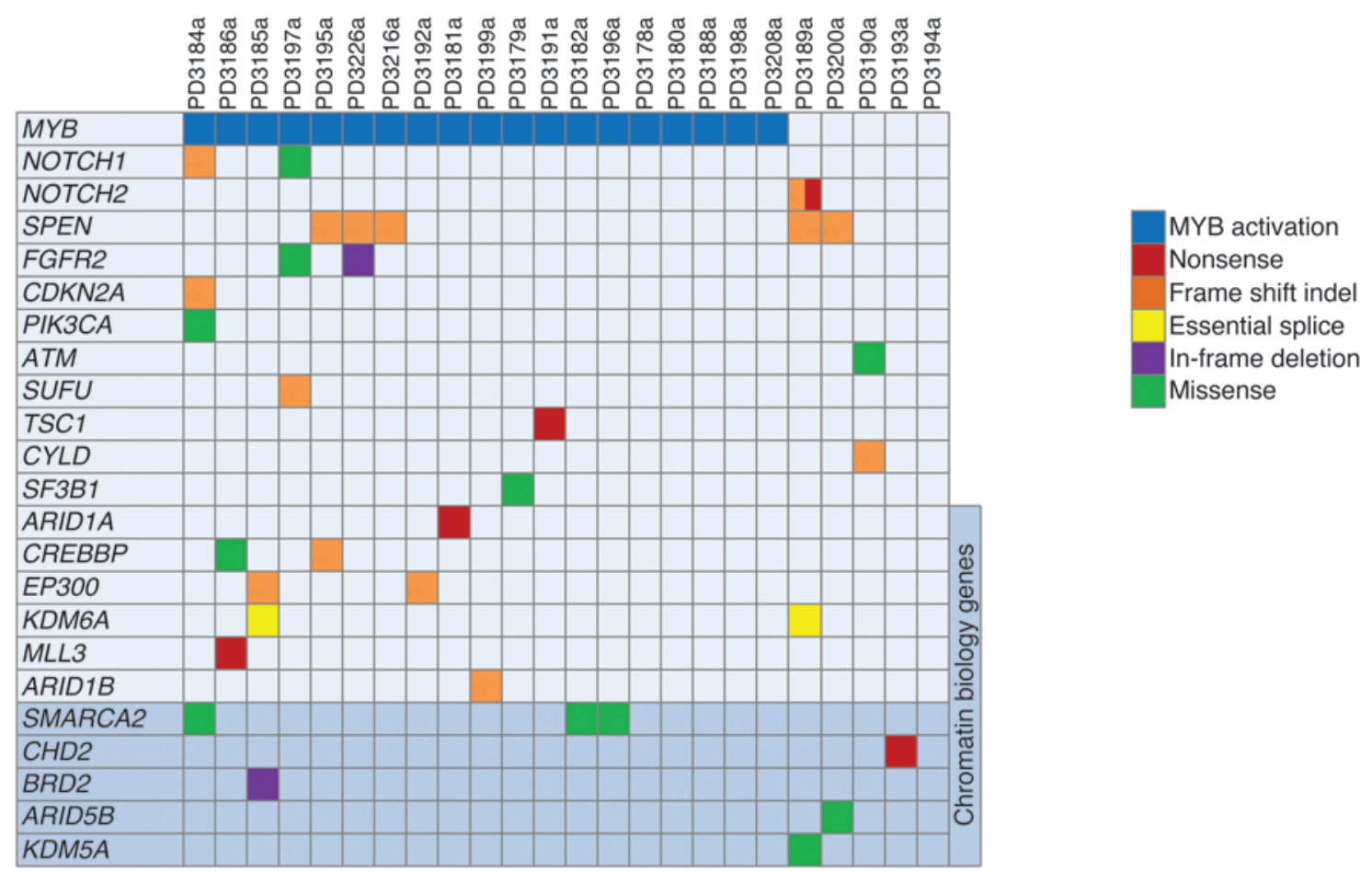

\section{Figure 1}

Somatic mutations identified in known cancer genes, SPEN, and genes involved in chromatin biology. The genes are listed on the left hand side and the adenoid cystic samples across the top. The darker shading at the bottom of the figure indicates genes involved in chromatin biology that have not previously been implicated in cancer.

fusion event. These data would be consistent with CDKN2A playing a role in a proportion of ACC. We also identified a canonical activating mutation in PIK3CA (p.H1047L) and a missense mutation in the ATM kinase (p.R337C). Arginine 337 is highly conserved and amino acid substitution mutations, p.R337S/H/C, have been reported in colorectal cancer and B cell chronic lymphocytic leukemia (1). The pattern of recurrence and multiple substitution is consistent with this mutation being likely oncogenic. Somatic truncating mutations were identified in $S U F U$, TSC1, and CYLD, 3 cancer genes associated with germline susceptibility to rare tumor syndromes as well as in the recently reported splicing factor cancer gene SF3B1 (7).

Somatic mutations were also identified in NOTCH1 and NOTCH2 in 3 cases. NOTCH1 had a missense (p.F1702S) and frameshift mutation (p.Y550fs*81) in 2 cases, while NOTCH2 had 2 truncating mutations (p.Q2308fs*5, p.E2420*) in a single case. Phe 1702 of NOTCH1 falls within a region frequently targeted in T-ALL for activating mutations, while the truncating mutation is upstream of reported activating truncations. Recent identification of frequent truncating mutations in head and neck cancers (head and neck squamous cell carcinoma [HNSCC]) in this same region suggested that $\mathrm{NOTCH1}$ can act as a tumor suppressor gene $(8,9)$. The presumptive compound heterozygous truncating NOTCH2 mutations in PD3189 are reminiscent of those reported in Hajdu-Cheney syndrome (MIM 102500) (10), which are suggested to be activating. Thus, the functional nature of NOTCH gene mutations in ACC is not easily discerned from the mutational pattern and may be different depending on the context of other cancer mutations in play.
Evidence was also found for alterations of genes involved in histone modification and chromatin remodeling previously implicated in cancer. Somatic mutations have been reported in ARID1A in clear cell ovarian, renal (ccRCC), transitional cell bladder (TCC) and gastric carcinoma (3, 11-13), CREBBP and EP300 in non-Hodgkin lymphoma (NHL) (14), and KDM6A (UTX) in ccRCC and TCC $(3,11)$. Each of these genes had only truncating mutations identified, with the exception of a truncating and missense mutation in CREBBP. The missense mutation, p.R1446C, occurs in the KAT11 domain and Arg1446 is exquisitely conserved through to plants, supporting its functional importance. Three previous substitution mutations at this residue have been reported (1). Like ATM, the recurrent targeting of the same amino acid for multiple substitutions suggests an oncogenic phenotype for these mutations. Truncating mutations were also identified in $M L L 3$, an H3K4 methylase with mutations previously reported in medulloblastoma and TCC $(11,15)$, and in ARID1B, a component of the switch/sucrose non-fermentable chromatin remodeling complex (SWI/SNF) complex like ARID1A with recurrent mutations recently identified in breast cancer (16). SMARCA2, which encodes the BRM ATPase subunit of the SWI/SNF complex was also implicated by 3 somatic missense mutations. Also likely affecting chromatin regulation were a truncating mutation in CHD2 (a chromodomain helicase ATPase), an in-frame deletion in BRD2 (a bromodomain protein which binds acetylated lysine residues of histone H4), missense mutations in ARID5B (an ARID domain family member implicated in childhood acute lymphoblastic leukemia; ref. 17) and KDM5A, an H3K4 demethylase and com- 

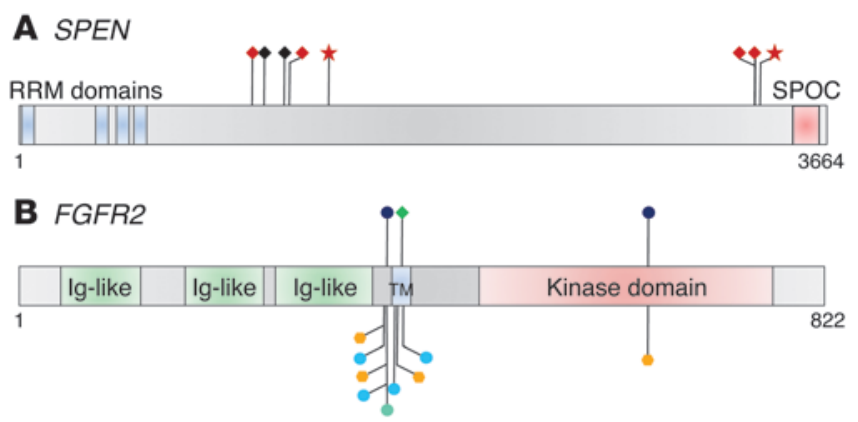

of SPEN through a further 42 cases identified 2 additional SPEN truncating mutations (p.R1403*, p.Q3355*), both in solid histology cases, thus confirming the importance of SPEN mutations in a proportion of ACC in general and in the poor prognosis solid histology subtype in particular. We were not able to assess $\mathrm{LOH}$ for these 2 cases. Recent work has identified truncating SPEN and NOTCH2 mutations in splenic marginal zone lymphoma (21). Of note, none of the SPEN mutations reported had evidence for loss of the wild-type allele, similarly arguing against a simple lossof-function mechanism for SPEN mutation in this cancer type. Given the identification of SPEN and NOTCH mutations in ACC, it is reasonable to suggest that deregulation of $\mathrm{NOTCH}$ signaling is playing a role in a proportion of cases. These data suggest an opportunity to explore NOTCH pathway targeting as a potential therapeutic angle in ACC.

Of particular interest from a potential therapeutic aspect was the identification of mutations in FGFR2, encoding a receptor tyrosine kinase for basic/acidic and keratinocyte growth factors. Two mutations, p.Y376C and p.I389_V393>M, were identified in PD3197a and PD3226a. The p.Y376C mutation is identical to that observed in Beare-Stevenson cutis gyrata syndrome (MIM 123790), one of the autosomal dominant craniosynostotic syndromes characterized by kinase-activating mutations in FGFR family members. Mutations at the paralogous residue in FGFR3, p.Y373C, give rise to thanatophoric dysplasia type I (MIM 187600). Somatic p.Y376C mutations have been reported in endometrial and ovarian carcinoma (ref. 1 and Figure 2B). This mutation and analogous substitutions have been shown to be activating through constitutive receptor dimerization via creating unpaired cysteine residues resulting in ligand-independent receptor activation $(22,23)$. The in-frame deletion occurs in the transmembrane domain and is likely similar by analogy with other activating mutations (22). Sequencing of FGFR2 through a further 25 cases identified a p.K642R mutation in the kinase domain identical to that described in Pfeiffer syndrome (MIM 101600), confirming the importance of FGFR2 activating mutations in a subset of ACC. There are currently several small molecule inhibitors of FGFRs in clinical trial. Indeed, a trial of one of these, Dovitinib, in ACC has recently opened (24). It would be of considerable interest to evaluate FGFR2 mutation status in tumors of participants.

In summary, systematic analyses of ACC genomics have uncovered the involvement of multiple cancer genes that are likely to be contributing to tumor development in the context of MYB activation. These data point to disruption of chromatin regulation as a major factor in ACC development. Further, identification of SPEN and NOTCH mutations implicate NOTCH signaling deregulation in a proportion of cases. Finally, these analyses have identified activating mutations of the receptor tyrosine kinase FGFR2 in a proportion of cases, thus suggesting a potential therapeutic point of attack on this rare but lethal cancer.

\section{Methods}

Diagnosis of ACC. The diagnosis of ACC was only made based on the presence in any part of the tumor of a tubular and/or cribriform patterns, regardless of the presence and extent of the solid component.

Assessment of MYB fusion status. MYB-NFIB fusion status was investigated using a combination of fusion transcript sequencing, qRT-PCR, FISH, and $3^{\prime}$ rapid amplification of cDNA ends (3' RACE), as described previously (25). The particular data supporting a scoring of MYB activation in any given sample are given in Supplemental Table 1. 
Exome sequencing and variant detection. See Supplemental Methods for details of exome enrichment, sequencing, variant detection, and validation. The sequence data have been deposited at the European Genomephenome Archive (EGA00001064049).

Detection of copy number variation. SNP array hybridization on the Affymetrix SNP6.0 platform was as per Affymetrix Protocols. Copy number analysis was performed using ASCAT (version 2.1) accounting for nonneoplastic cell admixture and tumor aneuploidy (26) and resulted in integer allelespecific copy number profiles for the tumor cells. The data have been deposited in Array Express (E-MTAB-1141).

Expression array analysis. See Supplemental Methods for details of the expression array analysis and SPEN expression by qRT-PCR. The expression array data have been deposited in Array Express (E-MTAB-1397).

Statistical assessment of the effects of histology and MYB status. A generalized linear model with negative binomial distributions was used to fit the number of mutations using the predictor variables (a) histological subtype and (b) MYB status. Neither variable was a significant predictor of the number of mutations ( $P$ values $=0.34$ and 0.28 , respectively). $P<0.05$ was considered significant.

Study approval. Collection and use of patient samples for this study was approved by the appropriate local IRB and further local research ethics committee (LREC) approval was obtained for this genomic study specifically. Patient samples were obtained under a waiver of informed consent from the IRB.

\section{Acknowledgments}

This work was supported by funding from the Adenoid Cystic Carcinoma Research Foundation and the Wellcome Trust (grant refer- ence $077012 / Z / 05 / Z$ ), the NIH National Institute of Dental and Craniofacial Research (NIDCR), and the NIH Office of Rare Diseases Research (ORDR) (grant number U01DE019765). S.M. Lippman and A.K. El-Naggar are supported by the NIH National Cancer Institute MD Anderson Cancer Center Head and Neck Specialized Program of Research Excellence (SPORE; P50 CA097007). P.J. Campbell is personally funded through a Wellcome Trust Senior Clinical Research Fellowship (grant reference WT088340MA). P. Van Loo is a postdoctoral researcher of the Research Foundation Flanders (FWO). A. Shlien is supported by the H.L. Holmes Award from the National Research Council Canada and an EMBO Fellowship. I. Varela is supported by a fellowship from The International Human Frontier Science Program Organization.

Received for publication October 9, 2012, and accepted in revised form April 11, 2013.

Address correspondence to: Adel K. El-Naggar, The Department of Pathology, The University of Texas MD Anderson Cancer Center, 1515 Holcombe Blvd., Unit 085, Houston, Texas 77030, USA. Phone: 713.792.3109; Fax: 713.745.1105; E-mail: anaggar@mdanderson.org. Or to: Andrew Futreal, Department of Genomic Medicine, The University of Texas MD Anderson Cancer Center, Unit 1954, P.O. Box 301429, Houston, Texas 77230, USA. Phone: 713.794.4764; Fax: 713.792.9636; E-mail: afutreal@mdanderson.org.

Andrew Futreal's present address is: Genomic Medicine, The University of Texas MD Anderson Cancer Center, Houston, Texas, USA.
1. Wellcome Trust Sanger Institute. Catalogue of somatic mutations in cancer. COSMIC Web site. http://www.sanger.ac.uk/genetics/CGP/cosmic/. Updated December 6, 2012. Accessed May 10, 2013.

2. Persson M, Andrén Y, Mark J, Horlings HM, Persson F, Stenman G. Recurrent fusion of MYB and NFIB transcription factor genes in carcinomas of the breast and head and neck. Proc Natl Acad Sci US A. 2009;106(44):18740-18744

3. Varela I, et al. Exome sequencing identifies frequent mutation of the SWI/SNF complex gene PBRM1 in renal carcinoma. Nature. 2011;469(7331):539-542.

4. Bhayani MK, et al. Prognosis and risk factors for early-stage adenoid cystic carcinoma of the major salivary glands. Cancer. 2011;118(11):2872-2878.

5. Rao PH, et al. Deletion of 1p32-p36 is the most frequent genetic change and poor prognostic marker in adenoid cystic carcinoma of the salivary glands. Clin Cancer Res. 2008;14(16):5181-5187.

6. Persson $\mathrm{M}$, et al. Clinically significant copy number alterations and complex rearrangements of MYB and NFIB in head and neck adenoid cystic carcinoma. Genes Chromosomes Cancer. 2012;51(8):805-817.

7. Papaemmanuil E, et al. Somatic SF3B1 mutation in myelodysplasia with ring sideroblasts. NEnglJ Med. 2011;365(15):1384-1395.

8. Agrawal N, et al. Exome sequencing of head and neck squamous cell carcinoma reveals inactivating mutations in NOTCH1. Science. 2011; 333(6046):1154-1157.
9. Stransky N, et al. The mutational landscape of head and neck squamous cell carcinoma. Science. 2011;333(6046):1157-1160.

10. Simpson MA, et al. Mutations in NOTCH2 cause Hajdu-Cheney syndrome, a disorder of severe and progressive bone loss. Nat Genet. 2011;43(4):303-305.

11. Gui Y, et al. Frequent mutations of chromatin remodeling genes in transitional cell carcinoma of the bladder. Nat Genet. 2011;43(9):875-878

12. Wang K, et al. Exome sequencing identifies frequent mutation of ARID1A in molecular subtypes of gastric cancer. Nat Genet. 2011;43(12):1219-1223.

13. Wiegand KC, et al. ARID1A mutations in endometriosis-associated ovarian carcinomas. NEnglJ Med. 2010;363(16):1532-1543.

14. Pasqualucci $\mathrm{L}$, et al. Inactivating mutations of acetyltransferase genes in B-cell lymphoma. Nature. 2011;471(7337):189-195.

15. Parsons DW, et al. The genetic landscape of the childhood cancer medulloblastoma. Science. 2011; 331(6016):435-439.

16. Stephens PJ, et al. The landscape of cancer genes and mutational processes in breast cancer. Nature. 2012; 486(7403):400-404.

17. Paulsson K, et al. Genetic landscape of high hyperdiploid childhood acute lymphoblastic leukemia. Proc Natl Acad Sci US A. 2010;107(50):21719-21724.

18. Oswald F, et al. SHARP is a novel component of the Notch/RBP-J[kappa] signalling pathway. EMBO J. 2002;21(20):5417-5426
19. Shi Y, et al. Sharp, an inducible cofactor that integrates nuclear receptor repression and activation. Genes Dev. 2001;15(9):1140-1151.

20. Li J, et al. The C terminus of MINT forms homodimers and abrogates MINT-mediated transcriptional repression. Biochim Biophys Acta. 2005; 1729(1):50-56.

21. Rossi $\mathrm{D}$, et al. The coding genome of splenic marginal zone lymphoma: activation of $\mathrm{NOTCH} 2$ and other pathways regulating marginal zone development. J Exp Med. 2012;209(9):1537-1551.

22. Wilkie AO, Patey SJ, Kan SH, van den Ouweland AM, Hamel BC. FGFs, their receptors, and human limb malformations: Clinical and molecular correlations. Am J Med Genet. 2002;112(3):266-278.

23. Byron SA, et al. FGFR2 mutations are rare across histologic subtypes of ovarian cancer. Gynecol Oncol. 2010;117(1):125-129.

24. University of Virginia. Study of Dovitinib (TKI258) in Adenoid Cystic Carcinoma (ACC). NIH Web site. http://clinicaltrials.gov/ct2/show/NCT01524692. Updated January 15, 2013. Accessed May 10, 2013.

25. Mitani Y, et al. Novel chromosomal rearrangements and break points at the $\mathrm{t}(6 ; 9)$ in salivary adenoid cystic carcinoma: association with MYB-NFIB chimeric fusion, MYB expression, and clinical outcome. Clin Cancer Res. 2011;17(22):7003-7014.

26. Van Loo P, et al. Allele-specific copy number analysis of tumors. Proc Natl Acad Sci U S A. 2010; 107(39):16910-16915. 